SLAC-PUB-9037

BABAR-PROC-01/70

hep-ex/0204001

November, 2001

\title{
Inclusive Semileptonic $B$ Decays at BABAR
}

\author{
U. Langenegger \\ Stanford Linear Accelerator Center \\ Stanford University, Stanford, CA 94309, USA \\ (for the BABAR Collaboration)
}

\begin{abstract}
We present measurements of the inclusive semileptonic branching fractions of charged and neutral $B$ mesons using $20.6 \mathrm{fb}^{-1}$ of data measured at the $\Upsilon(4 S)$ with the BABAR detector. Events are tagged with a fully reconstructed hadronic decay of a $B$ meson. The correlation between the flavor of the tag $B$ meson and the electron charge allows the separation of prompt semileptonic $B$ decays and cascade semileptonic charm decays. We obtain the preliminary inclusive semileptonic branching fraction of charged $B$ mesons $b_{+}=0.103 \pm 0.006 \pm 0.005$, neutral $B$ mesons $b_{0}=0.104 \pm 0.008 \pm 0.005$, their average $b=0.104 \pm 0.005 \pm 0.004$, and their ratio $b_{+} / b_{0}=0.99 \pm 0.10 \pm 0.03$.
\end{abstract}

Contributed to the Proceedings of the International Europhysics Conference on High Energy Physics (HEP 2001), 7/13/2001 - 7/18/2001, Budapest, Hungary

Stanford Linear Accelerator Center, Stanford University, Stanford, CA 94309

Work supported in part by Department of Energy contract DE-AC03-76SF00515. 


\section{Introduction}

In this paper we report a preliminary determination of the semileptonic branching fractions $b_{+}$and $b_{0}$ of charged and neutral $B$ mesons, respectively, obtained with measurements of electron] spectra in events tagged by a fully reconstructed hadronic decay of a $B$ meson. Electrons from the prompt semileptonic decay of the second $B$ meson are separated from electrons of cascade semileptonic charm decays using the correlation between the electron charge and the flavor of the $\operatorname{tag} B$ meson.

\section{Data sample and detector}

The data for this analysis were collected with the BABAR detector at the PEP-II asymmetric $e^{+} e^{-}$ collider [1] in 1999-2000 and amount to an integrated luminosity of $20.6 \mathrm{fb}^{-1}$ taken at the $\Upsilon(4 S)$.

The BABAR detector is a large-acceptance solenoidal spectrometer [2]. All components of the detector are used in the present analysis. Charged particles are detected and their momenta are measured with high precision in a five-layer double-sided silicon vertex tracker (SVT) and a 40layer drift chamber (DCH). Both SVT and DCH also provide ionization measurements used in charged particle identification. Additional charged particle identification information is obtained from the Cherenkov angle measurement in a detector of internally reflected Cherenkov light (DIRC). Electromagnetic showers of photons and electrons are measured with excellent resolution in the electromagnetic calorimeter (EMC), composed of $6580 \mathrm{CsI}(\mathrm{Tl})$ crystals. The iron flux return (IFR), used in the identification of muons, is segmented and instrumented with multiple layers of resistive plate chambers.

\section{Event selection}

We select multihadron events containing one fully reconstructed hadronic decay of a $B^{0}$ or $B^{+}$ candidate and at least one additional charged track. Continuum background consisting of nonresonant $e^{+} e^{-} \rightarrow q \bar{q}$ production $(q=u, d, s, c)$ is reduced by requiring the ratio of the second to the zeroth Fox-Wolfram moment [3] to be $R_{2}<0.5$.

Electron candidates must have a ratio of calorimeter energy to track momentum of $0.89<$ $E / p<1.2$. The profile of the energy deposit is required to be consistent with an electromagnetic shower, both in lateral [4] and azimuthal [5] extent. The ionization measurement in the DCH and the Cherenkov angle measured in the DIRC (if available) are required to be consistent with the electron hypothesis at the $3 \sigma$ level. Candidates that are not matched to an EMC cluster are retained if their $d E / d x$ measurement in the $\mathrm{DCH}$ is consistent with the electron hypothesis. The misidentification probability for hadrons faking an electron signature in the detector is measured in data with samples of pions, kaons and protons that have been identified by kinematic requirements with high purity.

Muon candidates must pass requirements on the measured and expected number of interaction lengths penetrated, the position match between the extrapolated track and IFR hits, and on the average and spread of the number of IFR hits per layer.

Kaon candidates are identified with $d E / d x$ measurements both in the SVT and DCH and the number of Cherenkov photons and Cherenkov angle measured in the DIRC.

\footnotetext{
${ }^{1}$ Charge-conjugation is implied throughout this note.
} 
A photon candidate consists of a localized energy maximum in the EMC with a lateral energy profile consistent with a photon shower and a minimum energy of $E>30 \mathrm{MeV}$. Pairs of photons measured in the EMC are constrained to the known $\pi^{0}$ mass if their invariant mass is $115<m_{\gamma \gamma}<$ $150 \mathrm{MeV}$ and if the sum of their energies is larger than $100 \mathrm{MeV}$.

Pairs of charged pions are combined to form $K_{S}^{0}$ candidates if their invariant mass is between 462 and $534 \mathrm{MeV}$. Neutral and charged kaons are combined with pions to form $K^{*}$ candidates, if their invariant mass is within $100 \mathrm{MeV}$ of the nominal $K^{*}$ mass. $\rho^{+}$and $\rho^{0}$ candidates are reconstructed in the decay modes $\pi^{+} \pi^{0}$ and $\pi^{+} \pi^{-}$, if their invariant mass is within $\pm 150 \mathrm{MeV}$ of the nominal $\rho$ mass. $a_{1}^{+}$candidates are formed by combining three charged pions, if their invariant mass is between 1.0 and $1.6 \mathrm{GeV}$ and the $\chi^{2}$ probability of a vertex fit of the $a_{1}^{+}$candidate is greater than $0.1 \%$.

$\bar{D}^{0}$ candidates are reconstructed in the decay channels $K^{+} \pi^{-}, K^{+} \pi^{-} \pi^{0}, K^{+} \pi^{+} \pi^{-} \pi^{-}$, and $K_{S}^{0} \pi^{+} \pi^{-}$. $D^{-}$candidates are formed in the $K^{+} \pi^{-} \pi^{-}$and $K_{S}^{0} \pi^{-}$decay modes. $D^{*-}$ candidates are reconstructed by combining a $\bar{D}^{0}$ candidate and a soft pion. The selection of $D^{*-}$ candidates requires the mass difference $\Delta m=m_{\bar{D}^{0} \pi^{-}}-m_{\bar{D}^{0}}$ to be within $\pm 3 \sigma$ of the nominal value. $D^{* 0}$ candidates are reconstructed by combining a $D^{0}$ candidate with a $\pi^{0}$ or a photon. $J / \psi$ candidates are reconstructed in the decay modes $e^{+} e^{-}$and $\mu^{+} \mu^{-}$where at least one decay daughter must be positively identified as a lepton. $J / \psi$ candidates must have an invariant mass of $2.95<m_{e^{+} e^{-}}<$ $3.14 \mathrm{GeV}$ and $3.06<m_{\mu^{+} \mu^{-}}<3.14 \mathrm{GeV}$, respectively. $\psi(2 S)$ candidates are reconstructed in the decay channels $\psi(2 S) \rightarrow e^{+} e^{-}, \mu^{+} \mu^{-}$and $\psi(2 S) \rightarrow J / \psi \pi^{+} \pi^{-}$.

$B^{0}$ candidates are formed by combining a $D^{(*)-}$ candidate with a $\pi^{+}, \rho^{+}$, or $a_{1}^{+}$. In addition, we reconstruct the decay channels $B^{0} \rightarrow J / \psi K^{(*) 0}$ and $B^{0} \rightarrow \psi(2 S) K^{(*) 0}$. $B^{+}$candidates are reconstructed by combining a $D^{(*) 0}$ candidate with a $\pi^{+}, \rho^{+}$, or $a_{1}^{+}$. Furthermore, the decay channels $B^{+} \rightarrow J / \psi K^{(*)+}$ and $B^{+} \rightarrow \psi(2 S) K^{(*)+}$ are reconstructed.

The selection of $B$ candidates is based on two nearly uncorrelated variables, the difference $\Delta E$ between the energy of the $B$ candidate and the beam energy in the $\Upsilon(4 S)$ center-of-mass frame, and the beam-energy substituted mass $m_{E S}=\sqrt{\left(s / 2+\mathbf{p} \cdot \mathbf{p}_{i}\right)^{2} / E_{i}^{2}-\mathbf{p}^{2}}$, where $\sqrt{s}$ is the center-of-mass energy, $E_{i}$ and $\mathbf{p}_{i}$ are the total energy and the momentum of the initial state in the laboratory frame, and $\mathbf{p}$ is the momentum of the $B$ candidate in the same frame. In the $\left(m_{E S}, \Delta E\right)$ plane a mode-dependent signal region is defined as $\left|m_{E S}-m_{E S}^{p e a k}\right|<3 \sigma\left(m_{E S}\right)$ and $\left|\Delta E-\Delta E^{\text {peak }}\right|<3 \sigma(\Delta E)$, where the resolutions $\sigma\left(m_{E S}\right)$ and $\sigma(\Delta E)$ are measured in data. If several $B$ candidates fulfill these requirements, only the one with the smallest $\left|\Delta E-\Delta E^{\text {peak }}\right|$ is retained. Signal and background yields are extracted from fits to the $m_{E S}$ distributions of $B$ candidates with a Gaussian distribution for the signal and a background given by [6]

$$
\frac{d N}{d m_{E S}} \propto m_{E S} \times \sqrt{1-\frac{m_{E S}^{2}}{E_{\text {beam }}^{* 2}}} \times \exp \left[-\zeta\left(1-\frac{m_{E S}^{2}}{E_{\text {beam }}^{* 2}}\right)\right],
$$

where the only free parameters are $\zeta$ and the normalization. In total, we reconstruct $7684 \pm$ $120_{\text {stat }} B^{+}$candidates and $6533 \pm 112_{\text {stat }} B^{0}$ candidates.

\section{Measurement of the inclusive semileptonic branching fractions}

In events with a reconstructed $\operatorname{tag} B$ candidate we search for electron candidates with a well measured track originating from the known beam-spot. Requirements on the momentum $p>$ $0.5 \mathrm{GeV}$ (both in the laboratory and center-of-mass frames) and the polar angle $23^{\circ}<\theta<135.9^{\circ}$ 
in the laboratory frame are applied to ensure good discrimination against hadrons and the full containment of the shower in the EMC. The mean electron identification efficiency is ( $89.8 \pm$ $\left.0.7_{\text {stat }}\right) \%$.

This electron spectrum is corrected for various background contributions as described below. Electrons from $J / \psi$ decays, photon conversions in the beam pipe and support structure of the SVT, and $\pi^{0} \rightarrow e^{+} e^{-} \gamma$ are detected using the invariant mass of an electron paired with a track of opposite charge. We correct the background spectrum for the inefficiency of the pair-finding algorithm and subtract the resulting spectrum from the total electron spectrum. The contribution of electrons from converted photons is sizeable in the lowest momentum bin, while electrons from $J / \psi$ decays make only a very small contribution at high momenta. The contribution from continuum events and combinatoric background in the reconstruction of the $\operatorname{tag} B$ meson is obtained with $B$ candidates in the $m_{E S}$ sideband and amounts to $4.2 \%$ of the total spectrum. The hadron fake contribution is estimated using misidentification probabilities for all hadronic tracks in tagged events. The mean hadron fake probability is $\left(0.25 \pm 0.005_{\text {stat }}\right) \%$ and is dominated by kaons. The fake contribution is largest at low momenta and amounts to $4.3 \%$ of the total electron spectrum.

Various processes other than prompt semileptonic $B$ decays contribute to the electron spectrum as well. Electrons from $\tau$ decays are estimated by Monte Carlo simulation, where a branching fraction of $\mathcal{B}(B \rightarrow \tau X)=2.6 \pm 0.4 \%$ is assumed [0]. Electrons from charm mesons originate either from $b \rightarrow c$ ("lower vertex" charm) or from $W^{-} \rightarrow \bar{c} s$ ("upper vertex" charm). The latter have the same charge as electrons from prompt $B$ decays and constitute a background, while the former are of opposite charge and are accounted for with the charge correlation to the flavor of the tag $B$ meson. Electrons from semileptonic "upper vertex" charm decays are estimated by Monte Carlo simulation, where the world average of the branching fractions for $B \rightarrow D_{s} X, B \rightarrow D^{(*)} \bar{D}^{(*)} K^{(*)}$, $D_{s} \rightarrow e X, D_{s} \rightarrow \tau X$, and semileptonic $D^{0}$ and $D^{+}$decays have been assumed. Semileptonic $B$ decays in the BABAR Monte Carlo simulation have been modeled according to the charm meson involved. A parametrization of HQET form factors, defined in [B], is used for $B \rightarrow D^{*} e \nu$, the model of Goity and Roberts [9] is used for the non-resonant decays $B \rightarrow D^{(*)} \pi e \nu$, whereas the ISGW2 model [10] is used for all other semileptonic decays.

The correlation between the charge of the electron and the flavor of the reconstructed tag $B$ meson allows the separation of the measured electron spectrum into contributions from prompt semileptonic $B$ decays and from cascade semileptonic charm decays (from the "lower vertex"). For events tagged with a $B^{0}$ meson, $B \bar{B}$ mixing must be taken into account with the $B_{d}$ mixing probability $\chi_{d}=0.174 \pm 0.009$ []]. In events tagged with a $B^{+}\left(B^{0}\right)$ candidate, we find $674 \pm 34_{\text {stat }}$ $\left(597 \pm 38_{\text {stat }}\right)$ prompt electrons. Figure 1 shows the spectra measured in events tagged with $B^{+}$and $B^{0}$ candidates. The prompt $B$ semileptonic branching fractions are derived from these numbers and the normalization (given by the number of reconstructed $B$ mesons) after correcting for (1) the difference in event selection efficiency between events where both $B$ mesons decay fully hadronically and events where one $B$ meson decays semileptonically, (2) the geometric acceptance of electron detection of $86.2 \%$, and (3) the extrapolation of the momentum spectrum of $(5.3 \pm 0.2) \%$.

The measured values of the branching fractions are for charged $B$ mesons $b_{+}=0.103 \pm 0.006 \pm$ 0.005 and for neutral $B$ mesons $b_{0}=0.104 \pm 0.008 \pm 0.005$. The ratio of the branching fractions is determined to be $b_{+} / b_{0}=0.99 \pm 0.10 \pm 0.03$. These results are consistent with and comparable in precision to [1]]. The average branching fraction $b=0.104 \pm 0.005 \pm 0.004$ is consistent with the many other measurements at the $\Upsilon(4 S)$ and LEP.

The systematic errors are dominated by three sources: First, the uncertainty in the track reconstruction efficiency together with the systematics of the cross-feed in the $B$-reconstruction 

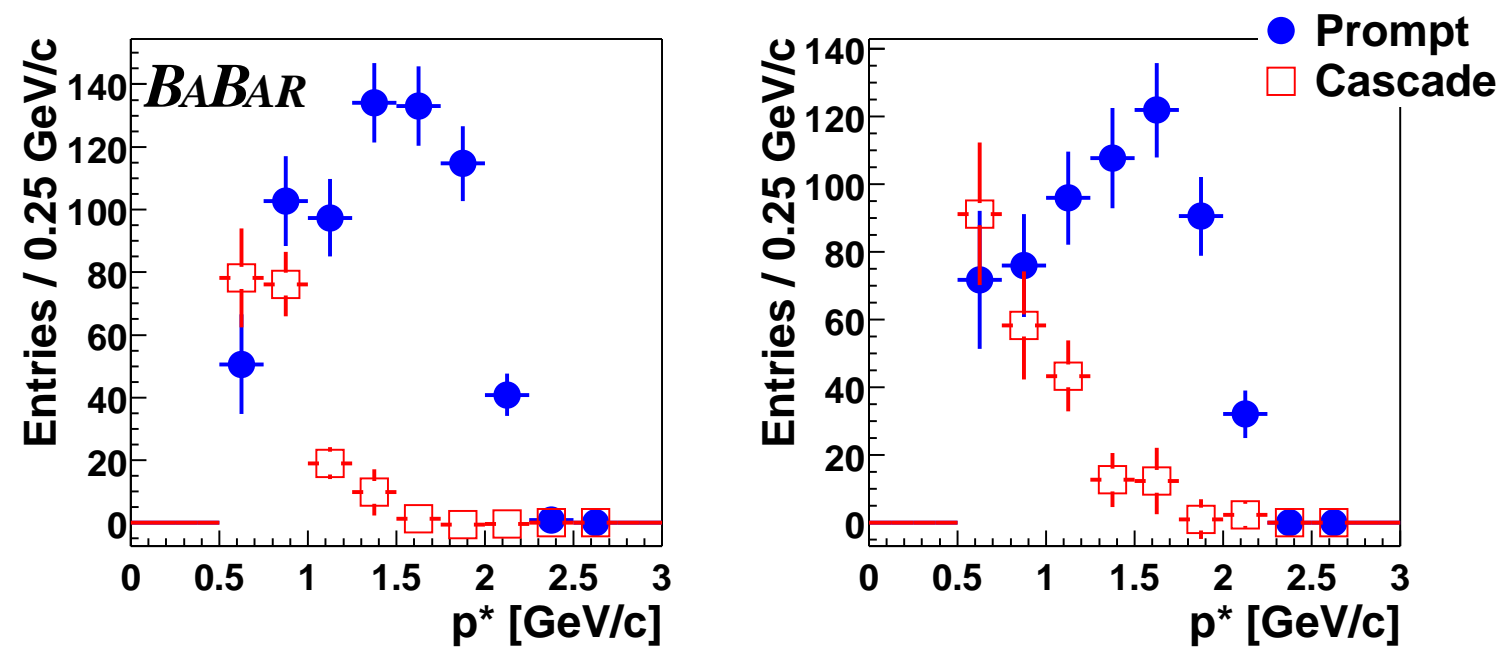

Figure 1: Measured electron spectra from $B \rightarrow X e \nu$ (filled circles) and from $b \rightarrow c \rightarrow Y e \nu$ (open squares) measured in events tagged with $B^{+}$(left) and $B^{0}$ (right) mesons.

amount to a total of $3.5 \%$. Second, electron identification (1.8\%) and the uncertainty in the hadron fake subtraction $(1.4 \%)$ have a significant contribution to the electron spectrum. The systematic errors associated with pair backgrounds from converted photons, Dalitz decays of $\pi^{0}$, and $J / \psi$ decays are negligible. Third, the uncertainty of physics processes contributing to the electron spectrum (mostly from upper vertex charm decays) amounts to $1.7 \%$. Only the first group of errors affects the determination of the ratio of branching fractions, the other two groups of errors cancel approximately.

\section{Conclusions}

We have presented a measurement of the inclusive semileptonic branching fractions of charged and neutral $B$ mesons in events tagged with a fully reconstructed hadronic decay of a $B$ meson. This analysis is statistics limited but already of comparable precision to the current world average. The systematic error can be improved with more statistics, leaving room for significant improvements as more data are accumulated at BABAR.

\section{References}

[1] PEP-II: An Asymmetric B Factory. Conceptual Design Report, SLAC-R-418 (1993).

[2] BABAR Collaboration, B. Aubert et al., hep-ex/0105044.

[3] G.C. Fox and S. Wolfram, Phys. Rev. Lett. 41 (1978) 1581.

[4] A. Drescher et al., Nucl. Instrum. Meth. A237 (1985) 464.

[5] R. Sinkus and T. Voss, Nucl. Instrum. Meth. A391 (1997) 360. 
[6] ARGUS Collaboration, H. Albrecht et al., Phys. Lett. B 241 (1990) 278.

[7] Particle Data Group, D.E. Groom et al., Eur. Phys. J. C 15 (2000) 1.

[8] CLEO Collaboration, J.E. Duboscq et al., Phys. Rev. Lett. 76 (1996) 3898.

[9] J.L. Goity and W. Roberts, Phys. Rev. D 51 (1995) 3459.

[10] D. Scora and N. Isgur, Phys. Rev. D 52 (1995) 2783.

[11] CLEO Collaboration, M. Artuso et al., Phys. Lett. B 399 (1997) 321. 